\title{
Quality-Factor Enhancing Locations for Substrate Mounted Resonators
}

\author{
Allen Anilkumar, Arun George and Gireesh Sharma N. \\ Inertial Systems Unit, Indian Space Research Organization, Trivandrum 695013, India.
}

(Received 22 January 2019; accepted 1 April 2020)

\begin{abstract}
An important but often overlooked factor that affects the performance of a meso/micro electro mechanical vibratory sensor is the structural interaction between the sensor's resonator and the substrate on which it is mounted. Situating resonators at node points eliminates this interaction and thereby helps to improve a resonator's quality-factor for a particular mode of vibration. This paper addresses the problem of locating a single degree of freedom springmass resonator on a generic cantilever substrate. The loci of natural frequencies obtained when the resonator's mounting location is varied are developed, and the nodal locations are identified. Thereafter a method to obtain these locations from the characteristic equation without solving the associated eigenvalue problem is described. Lookup tables detailing the nodal locations and the corresponding natural frequencies for various resonator parameters are presented. It is found that at these special nodal locations, the magnitude of the power transmitted through anchors is negligible, which ensures minimal structural interaction between the resonator and the substrate.
\end{abstract}

\section{INTRODUCTION}

Mechanical resonators are high performance devices designed to generate sinusoidal signals at precise frequencies. To excite these vibrations, energy is pumped into the device as sinusoidal forcing waves at the resonator's natural frequency. The resulting large amplitude resonant oscillations are subsequently utilized to generate the required signals.

The performance of micro/meso-scale resonators such as the ones used in Coriolis vibratory gyroscopes depends on the Qfactor of the resonators' operating mode of vibration. ${ }^{1,2}$ The accuracy of measurements made by such gyroscopes improves with an increase of its Q-factor and hence it is desirable to fabricate resonators with $\mathrm{Q}$-factors as high as possible. To realize high Q-factors, it is a common engineering practice to choose quartz based meso-scale resonators designs. ${ }^{3}$ Although the Qfactor associated with quartz is extremely high (reported to be over one billion ${ }^{3}$ ), in reality, several factors reduce the effective $\mathrm{Q}$-factor of a resonator mode. The various factors that affect the Q-factor of a resonator's vibratory mode are summarized in Table 1. The resultant Q-factor of the resonator's mode of operation can be expressed as a sum of the contributions from each of these factors in the following form: ${ }^{4}$

$$
\begin{aligned}
\frac{1}{Q}=\frac{1}{Q_{\text {material }}}+ & \frac{1}{Q_{\text {pressure }}} \\
& +\frac{1}{Q_{T E D}}+\frac{1}{Q_{\text {anchor }}}+\frac{1}{Q_{\text {other }}}
\end{aligned}
$$

Therefore the resultant Q-factor of a resonator mode is dictated by the factor with the least contributory Q-factor. Among these, the reduction of Q-factor due to improper anchorage of the resonator to its substrate motivates this work.

The type, the number and the location of the anchors attaching the resonator to a substrate significantly affects the Q-factor of the resonator's mode of vibration., 6 An improper anchor location leads to structural interaction between the resonator and the substrate which degrades resonator performance. Several researchers have investigated anchor induced reduction of Q-factor and have developed methods to predict the energy transmitted away from the resonator through the anchors. ${ }^{7-9}$ The energy carried by structural waves through the anchor into the substrate on which the resonator is mounted per cycle, is expressed for a cycle of harmonic motion with a period $T$ as:

$$
\pi_{L}=\frac{1}{T} \int_{0}^{T} \underline{F} \cdot \underline{\dot{u}} d t ;
$$

where $F$ is the vector of forces and moments acting at the anchor location and $\underline{\dot{u}}$ denotes the vector of velocities of the anchor point. ${ }^{10}$ The energy lost at an anchor depends on the velocity of the attachment point. Hence the obvious design strategy to be adopted to minimize anchor losses (and to maximize $Q_{\text {anchor }}$ ) is to locate the resonator such that the point of attachment is a node point (of the mode shape corresponding to the resonant frequency at which the resonator is designed to operate) of the entire assembly comprising the resonator as well as the substrate to which it is mounted.

Several researchers have investigated the direct problem of evaluating the vibratory characteristics of combined systems comprising masses elastically mounted to continuous media. Free vibrations of beams with elastically mounted masses has been explored in the past. ${ }^{11-14}$ Multi-span beams carrying multiple spring mass systems have also been investigated. ${ }^{15,16}$ Non-uniform beams carrying an arbitrary number of spring mass systems have also investigated ${ }^{17}$ as well. However, the inverse problem of estimating a design parameter, such as a stiffness or a mass, so that the combined system meets certain requirements has received less attention. A few notable inverse problems investigated include the imposition of nodes at certain locations on a continuous structure by attaching resonators, ${ }^{18-20}$ constructing a physically realizable system from a beam's eigen solutions ${ }^{21}$ and generating by design certain natural frequencies in a beam with an added mass. ${ }^{22}$ 
Table 1. Summary of the various factors that contribute to the final Q-factor of a resonator.

\begin{tabular}{||l|l|l||}
\hline Notation & $\begin{array}{l}\text { Contributing } \\
\text { factor }\end{array}$ & Description \\
\hline$Q_{\text {material }}$ & $\begin{array}{l}\text { Material fric- } \\
\text { tion }\end{array}$ & $\begin{array}{l}\text { The energy lost due to the friction in- } \\
\text { herent to the material constituting the } \\
\text { resonator }\end{array}$ \\
\hline$Q_{\text {pressure }}$ & $\begin{array}{l}\text { Viscosity of } \\
\text { the gaseous } \\
\text { medium }\end{array}$ & $\begin{array}{l}\text { The energy utilized to overcome the } \\
\text { resistance imparted by the gas sur- } \\
\text { rounding the resonator }\end{array}$ \\
\hline$Q_{T E D}$ & $\begin{array}{l}\text { Thermoelastic } \\
\text { effect }\end{array}$ & $\begin{array}{l}\text { The loss which arises due to the lo- } \\
\text { calized vibratory heating of a region } \\
\text { within the oscillating body }\end{array}$ \\
\hline$Q_{\text {anchor }}$ & Anchoring & $\begin{array}{l}\text { The energy transmitted from the res- } \\
\text { onator to the substrate through the an- } \\
\text { chors }\end{array}$ \\
\hline$Q_{\text {other }}$ & Others & $\begin{array}{l}\text { The dissipation due to other effects } \\
\text { such as surface defects and Akhiezer } \\
\text { effect }\end{array}$ \\
\hline
\end{tabular}

Table 2. Operating frequencies, Q-factors and damping levels for various high-Q resonators

\begin{tabular}{||l|l|l|l|l||}
\hline $\begin{array}{l}\text { Resonator } \\
\text { geometry }\end{array}$ & $\begin{array}{l}\text { Frequency } \\
\text { (MHz) }\end{array}$ & $\begin{array}{l}\text { Quality } \\
\text { factor, } \\
Q\end{array}$ & $\begin{array}{l}\text { Damping } \\
\text { factor, } \\
\zeta=\frac{1}{2 Q}\end{array}$ & $\begin{array}{l}\text { Refe- } \\
\text { rence }\end{array}$ \\
\hline Stemless disk & 74.3 & 98000 & $5.1 \mathrm{e}-6$ & 5 \\
\hline Disk & 0.247 & 100000 & $5 \mathrm{e}-6$ & 23 \\
\hline Hemispherical & 0.0184 & 143000 & $3.45 \mathrm{e}-6$ & 24 \\
\hline Dual ring & 19.7 & 150000 & $3.33 \mathrm{e}-6$ & 23 \\
\hline Cantilever & 0.0083 & 403000 & $1.24 \mathrm{e}-6$ & 24 \\
\hline Tuning fork & 0.0022 & 800000 & $6.25 \mathrm{e}-7$ & 25 \\
\hline Lame square beam & 6.35 & 1700000 & $2.94 \mathrm{e}-7$ & 26 \\
\hline
\end{tabular}

Unlike previous investigations, this work addresses the inverse problem of finding the locations on a substrate where an attached resonator experiences no structural interaction with its substrate for an intended operating resonant mode. A designer who aims to locate a resonator on a substrate with established natural frequencies, modes and nodal locations may perhaps be tempted to locate the resonator at the node points of the (bare) substrate. However, with the addition of the resonator, the location of the node points (of the assembly) deviate from those of the bare substrate. The deviation of the nodal locations upon attachment of the resonator is less significant for resonators with low mass and becomes substantial as the resonator mass increases. The location of the (new) node points of the assembly for a particular mode of vibration depends on the location of the resonator itself. The work explores the possibility of finding locations on a substrate where an attached resonator of a predetermined mass and operating frequency makes the same location a node point of the assembly comprising the substrate and the resonator. The investigation considers the problem of locating a generic single degree of freedom (DoF) spring-mass resonator on the commonly encountered cantilever substrate. The equation of motion governing the dynamics of such a resonator-substrate assembly is presented in Section 2. Thereafter, in Section 3, the natural frequencies and mode shapes of the system are obtained by solving the eigenvalue problem, and the nodal locations are identified from their loci. In Section 4 , a method is developed to calculate the nodal mounting locations from the characteristic equation without solving the eigenvalue problem. The nodal locations for various sets of resonator mass and stiffness are identified and tabulated.

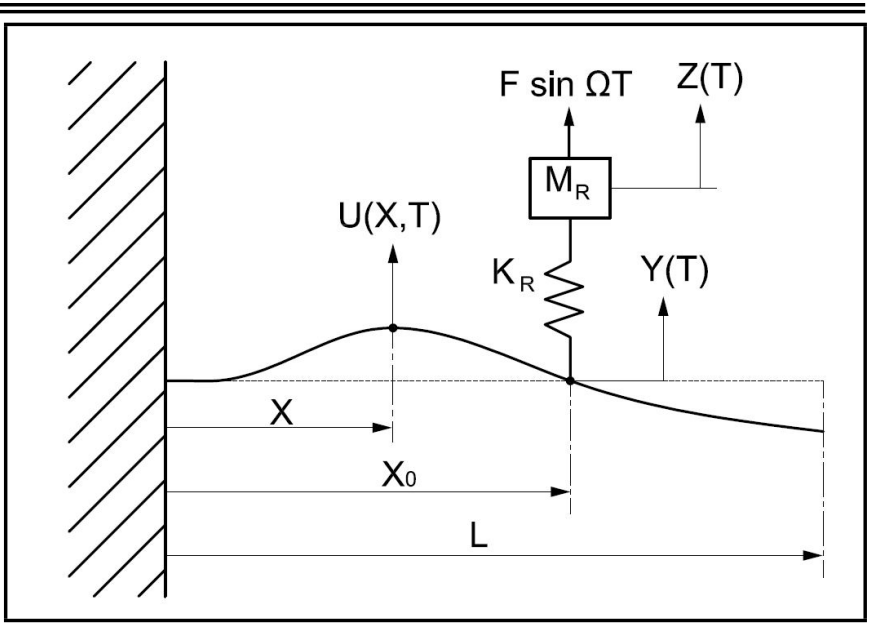

Figure 1. Schematic representation of a resonator mounted on a continuous substrate.

\section{THE MODEL}

The Q-factors and the associated damping factors ${ }^{1}$ for various high-Q resonators as reported in literature are presented in Table 2. It is evident that the damping factors associated with such high performance systems are extremely low. The time scale on which the resonator oscillations decay is much larger than the time scale associated with the resonator's oscillatory frequencies. The decay in the amplitude for each cycle can therefore be assumed to be negligible, and hence the vibratory characteristics of the high performance systems motivating this work is investigated in the following sections using an undamped structural model. The effect of damping is considered later in Section 5.

The system investigated here comprises a resonator mounted on a continuous elastic substrate. The resonator is modeled as a single-DoF spring-mass oscillator with a mass $M_{R}$ and a spring stiffness $K_{R}$ as represented in Fig. 1. The elastic substrate to which the resonator is mounted, is modeled as an Euler-Bernoulli beam ${ }^{27}$ of length $L$ with a flexural rigidity $E I$ (where $E$ is the modulus of elasticity of the homogeneous material constituting the beam and $I$ the area moment of inertia with respect to the neutral axis of bending) and a mass per unit length $\rho A$, where $\rho$ is the uniform material density and $A$ is the constant cross sectional area through out the length of the beam. The transverse deflection of the beam at a location $X$ along the length of the beam at any time $T$ is denoted by the variable $U(X, T)$. The resonator is assumed to be mounted at a location $X=X_{0}$. The displacement of mass $M_{R}$ is denoted by the variable $Z(T)$. In terms of the dimensionless variables, $x=\frac{X}{L}, u=\frac{U}{L}, z=\frac{Z}{L}, x_{0}=\frac{X_{0}}{L}, k=\frac{K_{R} L^{3}}{E I}, m=\frac{M_{R}}{\rho A L}$ and $t=T \sqrt{\frac{E I}{\rho A L^{4}}}$, the equations governing the motion of the system as presented in Reference ${ }^{12}$ are the following:

$$
\begin{gathered}
\frac{\partial^{4} u}{\partial x^{4}}+\frac{\partial^{2} u}{\partial t^{2}}=k(z-y) \delta\left(x-x_{0}\right) ; \quad x \in[0,1] \\
m \frac{d^{2} z}{d t^{2}}+k(z-y)=f \sin \Omega t .
\end{gathered}
$$

${ }^{1}$ For a single-DoF oscillator, the damping factor $\zeta$ and the $\mathrm{Q}$ are related as $Q=1 / 2 \zeta$. As $\zeta$ tends to zero, Q-factor tends to infinity. 
Here the variable $y=u\left(x_{0}, t\right)$ denotes the instantaneous displacement of the point of attachment of the resonator to the continuous substrate. The following boundary conditions are employed to model the clamped-free (cantilever) elastic substrate.

$$
\begin{aligned}
u(0, t) & =0 ; & \frac{\partial u}{\partial x}(0, t) & =0 ; \\
\frac{\partial^{2} u}{\partial x^{2}}(1, t) & =0 ; & \frac{\partial^{3} u}{\partial x^{3}}(1, t) & =0 .
\end{aligned}
$$

The equations presented above governing the dynamics of the system are solved and the solutions are examined in the following section.

\section{EIGEN STRUCTURE}

The Galerkin's method ${ }^{28,29}$ is employed to solve the above system of equations. Cantilever beam functions (denoted here by $\phi_{i}$ where $i=1 . . n$ ), obtained from the solution of the equation governing the transverse vibrations of a classic cantilever Euler-Bernoulli beam, ${ }^{27,29}$ are used as comparison functions to expand the variable $u$. Note that these functions satisfy the boundary conditions presented in Eq. (5). The expansion:

$$
u(x, t)=\sum_{i=1}^{n} \phi_{i}(x) \eta_{i}(t)
$$

is substituted in Eqs. (3) and (4), and the resulting expressions are multiplied $n$ times, each time by $\phi_{i}$ and integrated with respect to $x$ from $x=0$ to $x=1$. The obtained system of coupled ordinary differential equations, for $f=0$ can be expressed in the matrix form as:

$$
\underline{\underline{M}} \underline{\ddot{\eta}}+\underline{\underline{K}} \underline{\eta}=\underline{0}
$$

where the vector $\underline{\eta}$ is the transpose of the vector $\left\{\begin{array}{llllll}\eta_{1} & \eta_{2} & . & \eta_{n} & z\end{array}\right\}$. A general solution of the form:

$$
\underline{\eta}=\underline{u} e^{j \lambda t}
$$

is assumed and on substitution, Eq. (7) yields:

$$
\left(-\lambda^{2} \underline{\underline{M}}+\underline{\underline{K}}\right) \underline{u}=\underline{0}
$$

which is an algebraic eigenvalue problem, where $\lambda^{2}$ represents the eigenvalues and $\underline{u}$ the eigenvectors. The eigenvalue problem is solved; the results obtained are bench marked for certain parameter combinations in Appendix 1. The elements of the mass matrix $\underline{M}$ and the stiffness matrix $\underline{K}$ depend on the dimensionless system parameters $m, k$ and $\overline{x_{0}}$. The values of the resonator mass $m$ and stiffness $k$, depend on the designed resonator and is usually decided early in the product design cycle. For fixed values of $m$ and $k$ as the location $x_{0}$ changes, the natural frequencies, which are the the square roots of the eigenvalues, and the mode shapes (obtained from the eigenvectors using expansion (6)) of the system vary. At $x_{0}=0$ the natural frequencies of a standard clamped-free beam are recovered. In addition, a frequency $\omega_{r}=\sqrt{\frac{k}{m}}$ corresponding to the uncoupled resonator is also obtained. The loci of the first

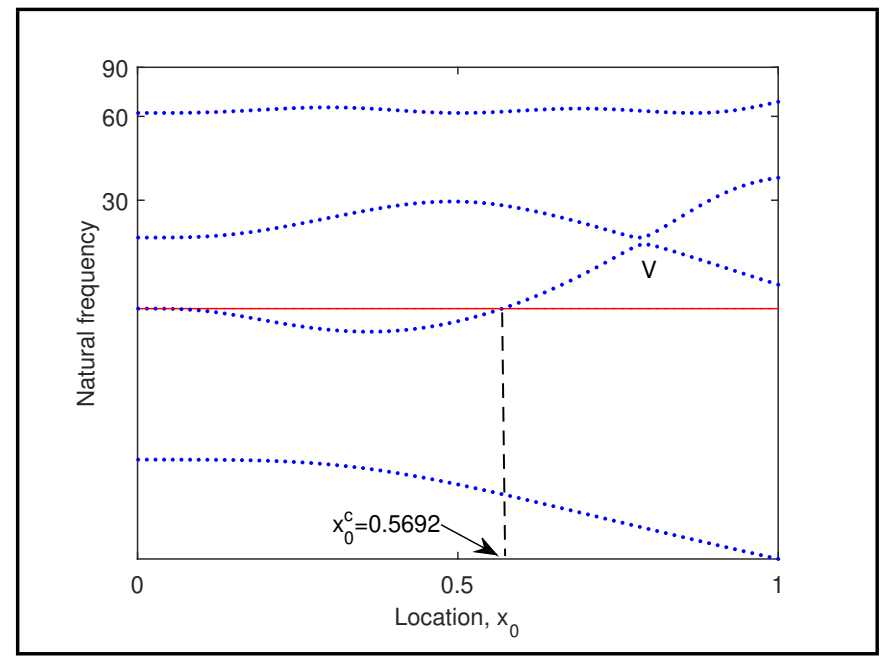

Figure 2. The loci of the lowest four natural frequencies as the attachment location $x_{0}$ is varied from 0 to 1 for mass $m=1$ and stiffness $k=150$. The (red) straight line parallel to the $x$ axis marks the resonator natural frequency $\omega_{r}=\sqrt{\frac{k}{m}}$.

four natural frequencies as the resonator attachment location is changed from $x_{0}=0$ to $x_{0}=1$, while keeping parameters $m=1$ and $k=150$ are presented in Fig. 2. It is observed that the variation of $x_{0}$ causes the various modes of the system to interact with each other. The loci presented in Fig. 2 show significant interaction between the second and the third mode of the system. The natural frequencies approach each other and veer away at the location corresponding to the point marked ' $\mathrm{V}$ '. Note that at $\mathrm{V}$, the mode shapes corresponding to these two natural frequencies are the same.

We search for the locations $x_{0}$ on the cantilever substrate where the attachment of a resonator of mass $m$ and stiffness $k$ makes $x_{0}$ a node point for a mode shape of the system. Such a nodal point would not oscillate if the system is forced at the frequency corresponding to that particular mode shape. For such a nodal location, $y(t)=0$ and Eq. (4) becomes:

$$
m \frac{d^{2} z}{d t^{2}}+k z=f \sin \Omega t .
$$

Equation (10) represents a single-DoF spring-mass oscillator with natural frequency $\sqrt{\frac{k}{m}}$, and which is forced at the frequency $\Omega$. Therefore, if $x_{0}$ is a nodal location, one natural frequency of the system modeled by Eq. (7) has to equal $\sqrt{\frac{k}{m}}$. Moreover, to excite this particular mode, for which $x_{0}$ is a node point, the forcing speed $\Omega$ has to equal $\sqrt{\frac{k}{m}}$. The nodal location $x_{0}^{c}$ is that value of $x_{0}$ at which any one of the natural frequencies equals $\sqrt{\frac{k}{m}}$. The frequency $\omega_{r}=\sqrt{\frac{k}{m}}$ is marked in Fig. 2 using the (red) solid line-type. The nodal location is obtained from the loci as the value of $x_{0}$ at which any locus intersects this horizontal line. The required nodal location for the pair of parameters $m=1$ and $k=150$ is found to be $x_{0}=0.5692$.

The loci of natural frequencies when location $x_{0}$ is varied for the case when $m=1$ and $k=300$ is presented in Fig. 3. The locus of the second mode intersects the frequency $\omega_{r}=\sqrt{\frac{k}{m}}$ at two distinct points, $x_{0}=0.6697$ and $x_{0}=0.9430$, and therefore there are two resonator mounting locations along the 


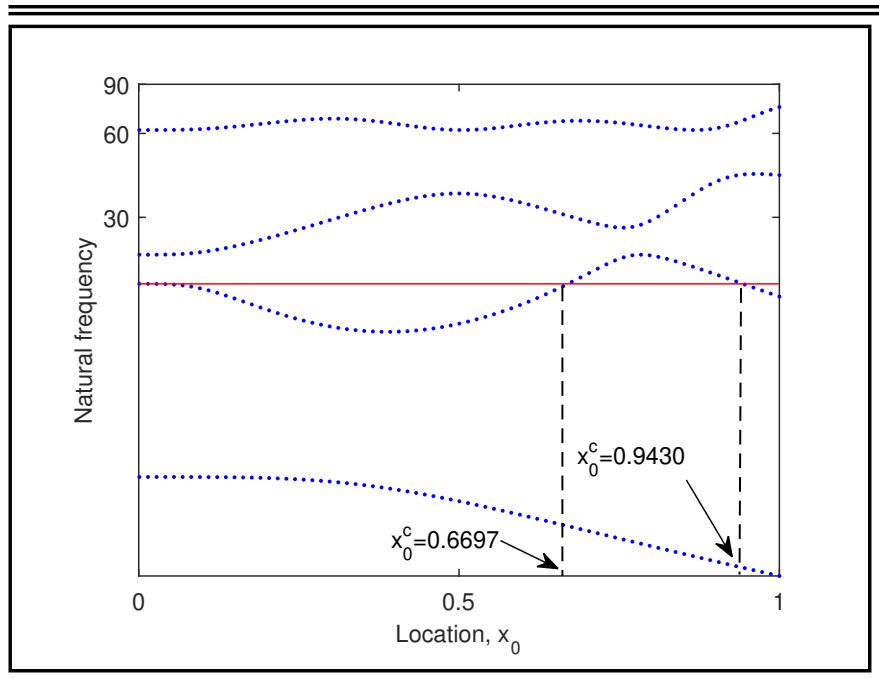

Figure 3. The loci of the lowest four natural frequencies as the attachment location $x_{0}$ is varied from 0 to 1 for mass $m=1$ and stiffness $k=300$. The (red) straight line parallel to the $x$ axis marks the resonator natural frequency $\omega_{r}=\sqrt{\frac{k}{m}}$.

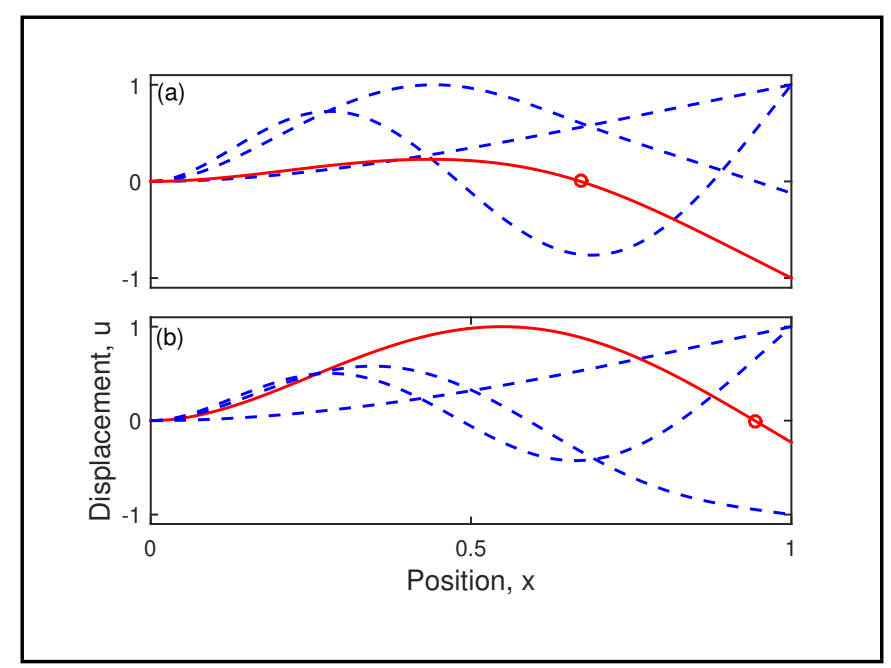

Figure 4. The first four mode shapes corresponding to the nodal locations marked on Fig. 3; (a) when $x_{0}^{c}=0.6697$ and (b) when $x_{0}^{c}=0.9430$.

substrate where the attachment point of the resonator to the substrate is a node point. The mode shapes corresponding to the lowest four natural frequencies of the system when the resonator is attached to these locations are presented in Fig. 4(a) and Fig. 4(b) respectively. The (red) solid line-type corresponds to the mode shape for which the attachment point of the resonator is a node point. The resonator locations are marked on these mode shapes with the (red) circle marker.

For resonator designs with parameters $m$ and $k$ that correspond to multiple possible nodal locations $x_{0}^{c}$ as presented in Fig. 3, the selection of one mounting location over the other is the designer's choice. However in most engineering cases, it is required that the oscillations of the resonator are of as high amplitude as possible when compared to the oscillations of the substrate. In other words, the total energy associated with the vibrating substrate (which is the sum of the kinetic and strain energies of the cantilever beam) should be as small as possible in comparison with the sum of the kinetic and potential energies of the resonator. A technique that can aid designers to choose one nodal location over the other is detailed in Ap-

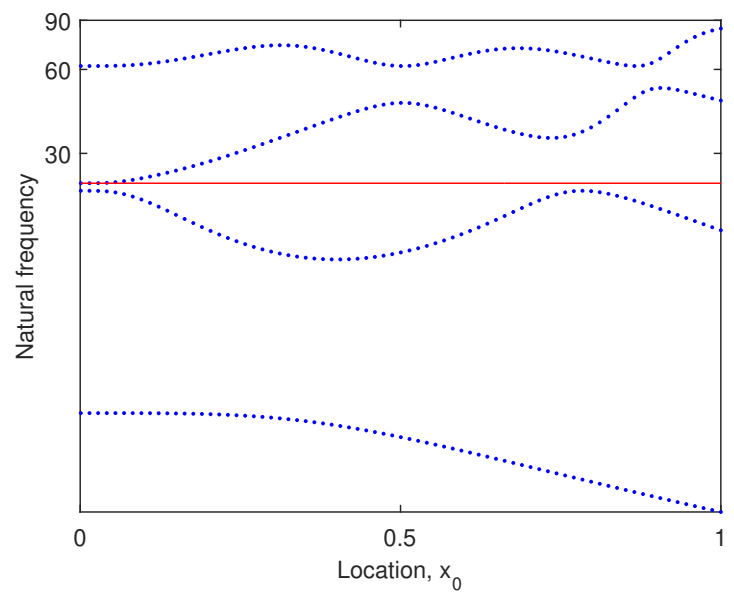

Figure 5. The loci of the lowest four natural frequencies as the attachment location $x_{0}$ is varied from 0 to 1 for mass $m=1$ and stiffness $k=550$. The (red) straight line parallel to the $x$ axis marks the resonator natural frequency $\omega_{r}=\sqrt{\frac{k}{m}}$.

\section{pendix 2.}

Figure 5 presents the loci of natural frequencies as location $x_{0}$ is varied for the values of $m=1$ and $k=550$. None of the loci crosses the line which marks the frequency $\omega_{r}=\sqrt{\frac{k}{m}}$. Therefore there are no locations $x_{0}$ along the beam where the attachment of a resonator with mass $m=1$ and stiffness $k=$ 550 makes the attachment point a node point.

\section{NODAL ATTACHMENT LOCATIONS}

The system under investigation has two points of fastening: the clamped boundary of the elastic substrate (at $x=0$ ) and the location $x_{0}$ where the resonator is attached to the substrate. The displacement $u(0, t)$ is zero due to the imposed boundary conditions. Hence for minimum anchor loss, the condition that needs to be satisfied in locating an already designed resonator, of predefined values $m$ and $k$, is to select $x_{0}$ such that it coincides with a node point of the combined assembly of the resonator and the substrate. A technique to obtain such nodal locations without tracing the loci of the natural frequencies is presented next.

The characteristic polynomial, associated with the eigenvalue problem presented in Eq. (9), is obtained by computing the determinant of the matrix $\left(-\lambda^{2} \underline{\underline{M}}+\underline{\underline{K}}\right)$ and is of degree $2(n+1)$. The characteristic equation is of the form:

$$
\begin{aligned}
& \lambda^{2(n+1)}+p_{(n+1)-1} \lambda^{2(n+1)-2} \\
& \quad+p_{(n+1)-2} \lambda^{2(n+1)-4}+\ldots+p_{0}=0
\end{aligned}
$$

For instance, if $n=3$ we obtain $\lambda^{8}+p_{3} \lambda^{6}+p_{2} \lambda^{4}+p_{1} \lambda^{2}+p_{0}=$ 0 . The $p_{i}$ of Eq. (11) are functions of the system parameters $m$, $k$ and $x_{0}$. For a particular resonator (with fixed values of $m$ and $k$ ), the only system parameter that the coefficients of the characteristic equation depend on is the anchor location $x_{0}$. At the nodal location, $x_{0}=x_{0}^{c}$, as explained earlier, one of the natural frequencies of the system must equal the uncoupled resonator frequency $\omega_{r}=\sqrt{\frac{k}{m}}$. To find the value of $x_{0}$ at which the attachment location of the resonator to the substrate becomes 


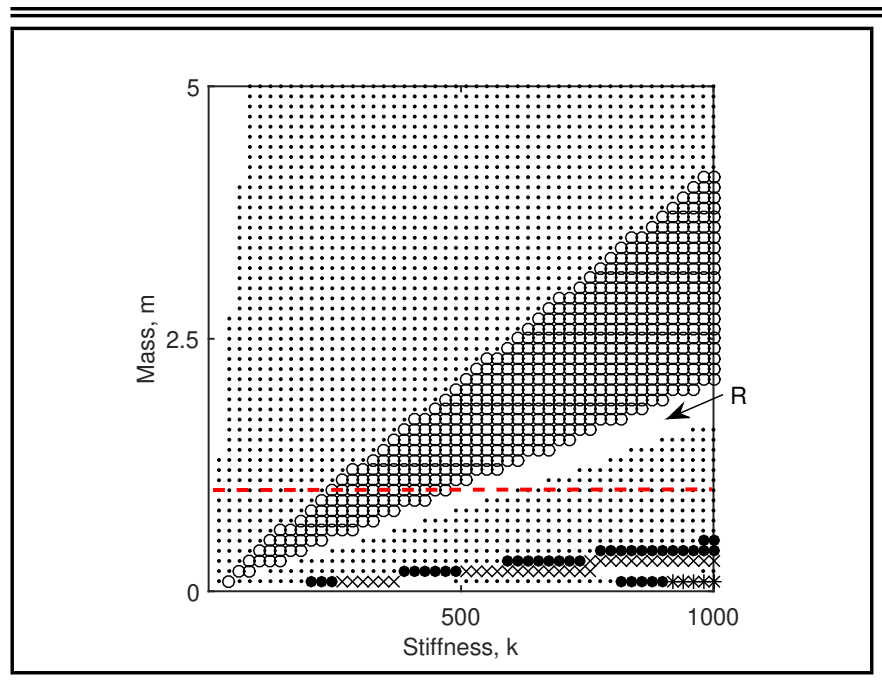

Figure 6. The number of possible nodal locations $x_{0}^{c}$ for a resonator of mass $m$ and stiffness $k$. The ' $'$ ' marker type represents parameter combinations with a single nodal location, the ' $\circ$ ' marker type with two, the ' $\bullet$ ' marker type with three, the ' $x$ ' marker type with four and the ' $*$ ' marker type with five. The unhatched regions correspond to parameter combinations without any possible nodal location $x_{0}^{c}$.

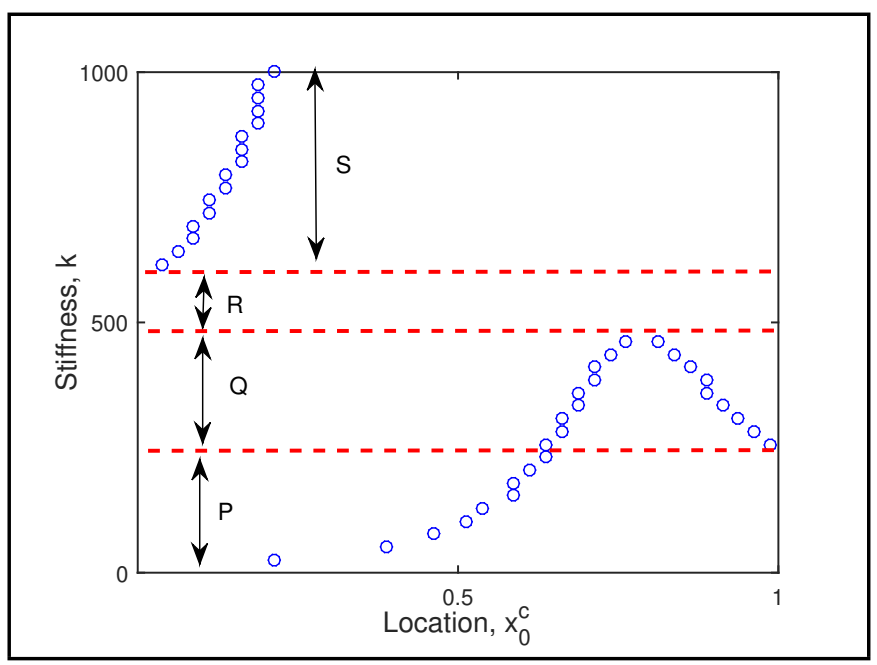

Figure 7. The variation of the nodal locations $x_{0}^{c}$ as stiffness $k$ changes for mass $m=1$. These parameters correspond to the (red) dashed line of Fig. 6 . Regimes $\mathrm{P}$ and $\mathrm{S}$ have one nodal location, $\mathrm{Q}$ has two and $\mathrm{R}$ has none.

a node point, the condition that one natural frequency should equal $\omega_{r}=\sqrt{\frac{k}{m}}$ is enforced. Therefore, $\lambda=\omega_{r}$ is substituted in the characteristic equation and the resulting nonlinear equation in $x_{0}$ is solved numerically in the domain $x_{0} \in[0,1]$ to obtain the required locations $x_{0}^{c}$. Here, the value of $x_{0}$ is computed using a combination of the bisection and the secant numerical methods.

As was seen in Section 3, it is possible that there exists multiple solutions for $x_{0}^{c}$ (i.e. multiple nodal locations) for a resonator of mass $m$ and stiffness $k$. The number of such nodal locations depends on the values of these resonator parameters. In general, as the value of the frequency $\omega_{r}$ increases, the number of possible solutions for $x_{0}^{c}$ within the regime $0<x<1$ also increases. A chart depicting the number of possible values of $x_{0}^{c}$ for various combinations of parameters $m$ and $k$ is presented in Fig. 6. The hatched regions represent parameter combinations with at least one possible value of $x_{0}$. There is
Table 3. Nodal locations and the lowest four natural frequencies for various resonator stiffnesses when resonator mass $m=0.05$. The frequency corresponding to the mode for which the resonator attachment location is a node point, is underscored.

\begin{tabular}{|c|c|c|c|c|c||}
\hline Stiffness & $\begin{array}{c}\text { Nodal } \\
\text { locations }\end{array}$ & \multicolumn{4}{|c|}{ Natural frequencies } \\
\hline$k$ & $x_{0}^{c}$ & $\omega_{1}$ & $\omega_{2}$ & $\omega_{3}$ & $\omega_{4}$ \\
\hline 1 & 0.1460 & 3.5149 & $\underline{\mathbf{4 . 4 7 2 1}}$ & 22.0371 & 61.7024 \\
\hline 10 & 0.6118 & 3.4341 & $\underline{\mathbf{1 4 . 1 4 2 1}}$ & 22.5181 & 61.7886 \\
\hline 50 & 0.2024 & 3.5145 & 21.6437 & $\underline{\mathbf{3 1 . 6 2 2 8}}$ & 62.4997 \\
\hline \multirow{3}{*}{150} & 0.4301 & 3.4921 & 20.8560 & $\underline{\mathbf{5 4 . 7 7 2 3}}$ & 64.9889 \\
\cline { 2 - 6 } & 0.5801 & 3.4506 & 21.1161 & $\underline{\mathbf{5 4 . 7 7 2 3}}$ & 65.0191 \\
\cline { 2 - 6 } & 0.8111 & 3.3370 & 22.0015 & $\underline{\mathbf{5 4 . 7 7 2 3}}$ & 64.2048 \\
\cline { 2 - 6 } & 0.9543 & 3.2411 & 20.8259 & $\underline{\mathbf{5 4 . 7 7 2 3}}$ & 70.2207 \\
\hline 300 & 0.1241 & 3.5158 & 21.9894 & 60.0485 & $\underline{\mathbf{7 7 . 4 5 9 6}}$ \\
\hline
\end{tabular}

an interesting unhatched region (marked R) banked by hatched regions on both sides. For combinations of values of $m$ and $k$ within this region, there are no locations $x_{0}$ along the substrate where the resonator can be mounted so that the attachment point is a node point for any mode shape of the system. The (red) dashed line corresponds to parameter $m=1$. For this value of $m$, there are various regimes of stiffnesses within $0<k<1000$ for which the nodal location $x_{0}$ can have either no solution, one solution or two solutions. These nodal locations as a function of stiffness $k$ are presented in Fig. 7. The stiffness values corresponding to regions marked $\mathrm{P}$ and $\mathrm{S}$ have one possible nodal location $x_{0}^{c}$, $\mathrm{Q}$ has two and $\mathrm{R}$ has none. The parameters chosen to develop Figs. 2, 3 and 5 fall in the regions marked P, Q and R of Fig. 7, respectively.

The nodal attachment locations $x_{0}^{c}$ for various values of $k$ when $m=0.05$ is presented in Table 3. For $k=1,10,50$ and 300 , the system has one nodal point each. However, for $k=150$, there are four possible attachment locations at which the mounting point is a node point for the assembly comprising the resonator and the substrate. The table also presents the lowest four natural frequencies of the system for each of these combinations of mass $m$ and stiffness $k$.

The nodal locations and natural frequencies for the cases when $m=0.1,0.25,0.5,1$ and 2 are presented in Tables 4, 5, 6, 7, and 8 respectively. As evident in Figs. 5, 6 and 7, a nodal location $x_{0}^{c}$ does not exist for all combinations of resonator parameters $m$ and $k$. For those values of $m$ and $k$, which do not yield a nodal location $x_{0}^{c}$, the corresponding table entries for $x_{0}^{c}$ are marked ' $x$ ' and the natural frequencies are left blank.

Throughout this investigation, the number of basis functions $n$ was taken to be 6 . The resulting system had 7 natural frequencies ( 6 of which originated from the previously mentioned basis functions $\phi_{i}$ and 1 from the resonator DoF) out of which the lowest four changed by less than 0.0005 percent when $n$ was increased from 5 to 6 . For instance, when $m=1$ and $k=150$, as $n$ was incremented from 5 to 6 , the value of $x_{0}^{c}$ changed negligibly and the fourth natural frequency, $\omega_{4}$ decreased by just 0.0002 percent from 62.3425 to 62.3423 .

\section{POWER TRANSMITTED THROUGH THE ANCHOR}

The energy transmitted per second through the anchors is a measure of the structural interaction between a resonator and 
Table 4. Nodal locations and the lowest four natural frequencies for various resonator stiffnesses when resonator mass $m=0.1$. The frequency corresponding to the mode for which the resonator attachment location is a node point, is underscored.

\begin{tabular}{|c|c|c|c|c|c||}
\hline \multirow{2}{*}{ Stiffness } & $\begin{array}{c}\text { Nodal } \\
\text { locations }\end{array}$ & \multicolumn{4}{|c||}{ Natural frequencies } \\
\hline$k$ & $x_{0}^{c}$ & $\omega_{1}$ & $\omega_{2}$ & $\omega_{3}$ & $\omega_{4}$ \\
\hline 1 & $\times$ & & & & \\
\hline 10 & 0.5042 & 3.4246 & $\underline{\mathbf{1 0 . 0 0 0 0}}$ & 22.6038 & 61.6970 \\
\hline 50 & $\times$ & & & & \\
\hline 150 & 0.2937 & 3.5038 & 20.5303 & $\underline{\mathbf{3 8 . 7 2 9 8}}$ & 65.9878 \\
\hline \multirow{3}{*}{300} & 0.4301 & 3.4685 & 19.8956 & $\underline{\mathbf{5 4 . 7 7 2 3}}$ & 67.8976 \\
\cline { 2 - 6 } & 0.5800 & 3.3885 & 20.3772 & $\underline{\underline{\mathbf{5 4 . 7 7 2 3}}}$ & 67.9836 \\
\cline { 2 - 6 } & 0.8111 & 3.1829 & 21.9757 & $\underline{\mathbf{5 4 . 7 7 2 3}}$ & 66.4487 \\
\cline { 2 - 6 } & 0.9543 & 3.0214 & 20.0900 & $\underline{\mathbf{5 4 . 7 7 2 3}}$ & 77.3137 \\
\hline
\end{tabular}

Table 5. Nodal locations and the lowest four natural frequencies for various resonator stiffnesses when resonator mass $m=0.25$. The frequency corresponding to the mode for which the resonator attachment location is a node point, is underscored.

\begin{tabular}{|c|c|c|c|c|c||}
\hline Stiffness & $\begin{array}{c}\text { Nodal } \\
\text { locations }\end{array}$ & \multicolumn{4}{|c|}{ Natural frequencies } \\
\hline$k$ & $x_{0}^{c}$ & $\omega_{1}$ & $\omega_{2}$ & $\omega_{3}$ & $\omega_{4}$ \\
\hline 1 & $\times$ & & & & \\
\hline 10 & 0.3242 & 3.4547 & $\underline{\mathbf{6 . 3 2 4 6}}$ & 22.3541 & 61.8747 \\
\hline 50 & 0.6118 & 3.1569 & $\underline{\mathbf{1 4 . 1 4 2 1}}$ & 24.3135 & 62.1638 \\
\hline 150 & $\times$ & & & & \\
\hline 300 & 0.2445 & 3.5005 & 19.5896 & $\underline{\mathbf{3 4 . 6 4 1 0}}$ & 67.9733 \\
\hline
\end{tabular}

Table 6. Nodal locations and the lowest four natural frequencies for various resonator stiffnesses when resonator mass $m=0.5$. The frequency corresponding to the mode for which the resonator attachment location is a node point, is underscored.

\begin{tabular}{||c|c|c|c|c|c||}
\hline Stiffness & $\begin{array}{c}\text { Nodal } \\
\text { locations }\end{array}$ & \multicolumn{4}{|c|}{ Natural frequencies } \\
\hline$k$ & $x_{0}^{c}$ & $\omega_{1}$ & $\omega_{2}$ & $\omega_{3}$ & $\omega_{4}$ \\
\hline 1 & $\times$ & & & & \\
\hline 10 & 0.1460 & 3.5048 & $\underline{\mathbf{4 . 4 7 2 1}}$ & 22.0646 & 61.7514 \\
\hline 50 & 0.5042 & 3.1208 & $\underline{\mathbf{1 0 . 0 0 0 0}}$ & 24.7245 & 61.6970 \\
\hline & 0.6697 & 2.7560 & $\underline{\mathbf{1 7 . 3 2 0 5}}$ & 26.9796 & 64.0120 \\
\cline { 2 - 6 } & 0.9430 & 2.1228 & $\underline{\mathbf{1 7 . 3 2 0 5}}$ & 35.2021 & 63.7346 \\
\hline 300 & $\times$ & & & & \\
\hline
\end{tabular}

Table 7. Nodal locations and the lowest four natural frequencies for various resonator stiffnesses when resonator mass $m=1$. The frequency corresponding to the mode for which the resonator attachment location is a node point, is underscored.

\begin{tabular}{|c|c|c|c|c|c||}
\hline Stiffness & $\begin{array}{c}\text { Nodal } \\
\text { locations }\end{array}$ & \multicolumn{4}{|c||}{ Natural frequencies } \\
\hline$k$ & $x_{0}^{c}$ & $\omega_{1}$ & $\omega_{2}$ & $\omega_{3}$ & $\omega_{4}$ \\
\hline 1 & $\times$ & & & & \\
\hline 10 & $\times$ & & & & \\
\hline 50 & 0.3730 & 3.1862 & $\underline{\mathbf{7 . 0 7 1 1}}$ & 24.0248 & 62.3493 \\
\hline 150 & 0.5692 & 2.6426 & $\underline{\mathbf{1 2 . 2 4 7 4}}$ & 28.7684 & 62.3423 \\
\hline & 0.6697 & 2.3438 & $\underline{\mathbf{1 7 . 3 2 0 5}}$ & 30.4062 & 66.4971 \\
\cline { 2 - 6 } 300 & 0.9430 & 1.6622 & $\underline{\mathbf{1 7 . 3 2 0 5}}$ & 42.7217 & 66.7933 \\
\hline
\end{tabular}

Table 8. Nodal locations and the lowest four natural frequencies for various resonator stiffnesses when resonator mass $m=2$. The frequency corresponding to the mode for which the resonator attachment location is a node point, is underscored.

\begin{tabular}{|c|c|c|c|c|c||}
\hline Stiffness & $\begin{array}{c}\text { Nodal } \\
\text { locations }\end{array}$ & \multicolumn{4}{|c||}{ Natural frequencies } \\
\hline$k$ & $x_{0}^{c}$ & $\omega_{1}$ & $\omega_{2}$ & $\omega_{3}$ & $\omega_{4}$ \\
\hline 1 & $\times$ & & & & \\
\hline 10 & $\times$ & & & & \\
\hline 50 & 0.2084 & 3.3905 & $\underline{\mathbf{5 . 0 0 0 0}}$ & 22.5040 & 62.3368 \\
\hline 150 & 0.4531 & 2.6377 & $\underline{\mathbf{8 . 6 6 0 3}}$ & 28.8844 & 62.1052 \\
\hline 300 & 0.5692 & 2.2087 & $\underline{\mathbf{1 2 . 2 4 7 4}}$ & 33.7125 & 63.0963 \\
\hline
\end{tabular}
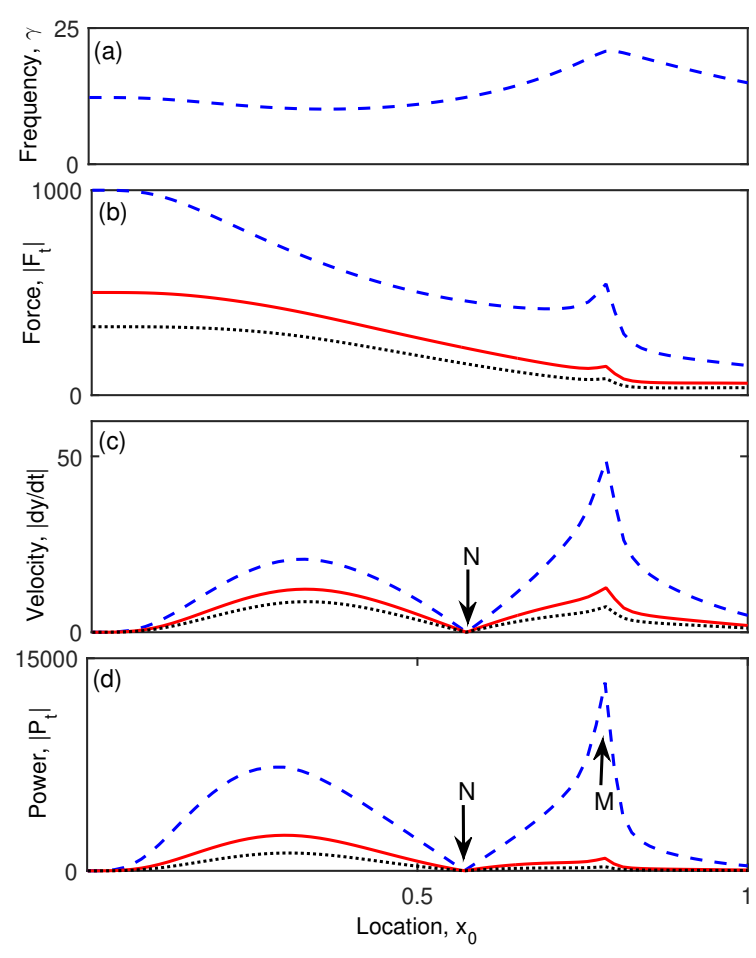

Figure 8. (a) The frequency of forcing, and the magnitudes of (b) the force transmitted through the anchor, of (c) the velocity of the anchorage location and of (d) the power transmitted by the force, as the attachment location of the resonator on the substrate is varied from $x_{0}=0$ to $x_{0}=1$ for three different sets of damping coefficients $\alpha=0.0122, \beta=0$ (marked by the dashed line type and corresponds to a Q-factor of 1000 for the mode under forcing), $\alpha=0.0122, \beta=8.165 e-5$ (marked by the solid line type and corresponds to a Q-factor of 500), and $\alpha=0.0122, \beta=1.633 e-4$ (marked by the dot line type and corresponds to a Q-factor of 333).

the substrate on which it is mounted. It is important for the performance of vibratory sensors, such as the ones motivating this investigation, that this interaction is minimal. The ideal anchoring location is the value of $x_{0}$ for which the amplitude of the power transmitted through the anchor is minimum. The effect of the attachment location $x_{0}$ on the force and the power transmitted through the point of anchorage (of the resonator to the substrate) is investigated here.

Vibratory sensors are operated at the resonance corresponding a mode of interest. Here we select the forcing speed $\gamma$ to be equal to the second natural frequency of the system. Note that $\gamma$ changes with the attachment location $x_{0}$ as presented in Fig. 8(a). The parameters $m=1$ and $k=150$ are selected (the loci of natural frequencies for the same set parameters was presented in Fig. 2). Note that the undamped, unforced variant of the same discretized system was investigated previously in Sections 3 and 4. A lightly dissipative version of the system presented in Section 2 is explored here. Damping is introduced directly in the discretized governing equations (Eq. (7)) and is assumed to be of the proportional type. ${ }^{30}$ The equations governing the forced system are obtained as:

$$
\underline{\underline{M}} \underline{\ddot{\eta}}+\underline{\underline{C}} \underline{\dot{\eta}}+\underline{\underline{K}} \underline{\eta}=\underline{Q} \sin \gamma t
$$

where $Q$ (which is of size $(n+1,1)$ ) is a matrix of zeros except the $n+1^{\text {th }}$ element which is one, and $\underline{\underline{C}}=\alpha \underline{\underline{M}}+\beta \underline{\underline{K}}$ where $\alpha$ and $\beta$ are scalar constants. The viscous damping 


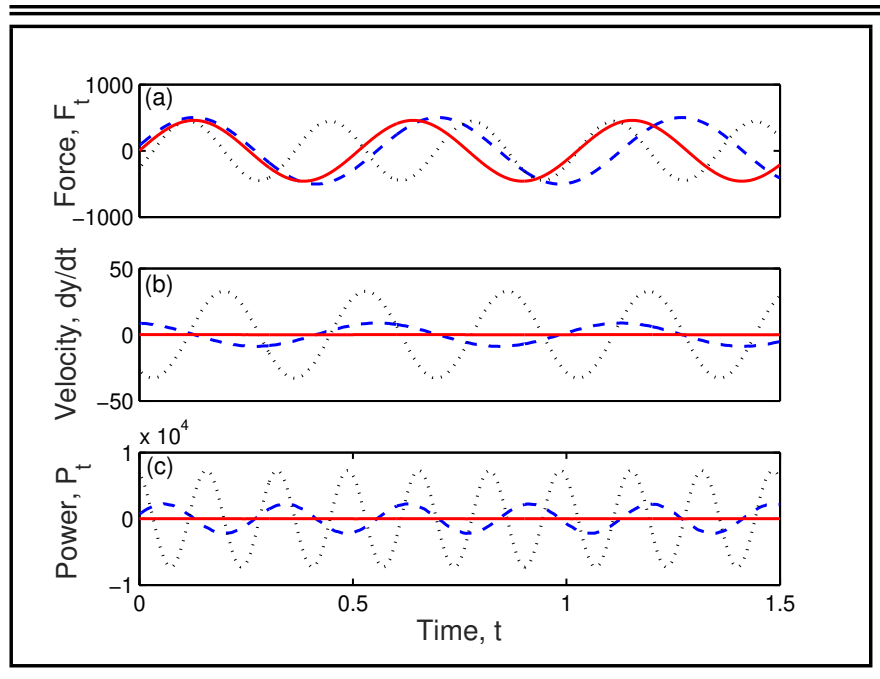

Figure 9. (a) The instantaneous force transmitted through the anchor, (b) the velocity of the anchor, and (c) the rate of work done by the force when the resonator attachment locations are $x_{0}=0.5$ (dash line-type), $x_{0}=0.5692$ (solid line-type) and $x_{0}=0.75$ (dot line-type) as functions of time when $m=1, k=150, \alpha=0.0122, \beta=0$ and $f=1$.

(c) experienced by mass $m$ (in the dimensionless form) is the $(n+1)^{t h}$ diagonal element of the damping matrix, i.e. $c=\underline{\underline{C}}(n+1, n+1)$. The solution $\underline{\eta}(t)$ of Eq. (12) is obtained by numerical integration using the ode 45 routine of MATLAB, and is mapped back to coordinates $u(x, t), y(t)$ and $z(t)$ using Eq. (6). The instantaneous force transmitted through the anchor is:

$$
F_{t}(t)=k(z-y)+c\left(\frac{d z}{d t}-\frac{d y}{d t}\right)
$$

The forces transmitted through the anchor as functions of time for three different anchorage locations $x_{0}=0.5,0.5692$ and 0.75 are presented in Fig. 9(a) when the constants of proportional damping take on values $\alpha=0.0122$ and $\beta=0$ (which correspond to a Q-factor of approximately 1000 for the mode of forcing). The velocity of the attachment points $\frac{d y}{d t}$ for these locations are presented in Fig. 9(b). The location $x_{0}=0.5692$ is a node point for the undamped version of the system and the introduced nominal damping has negligible effect on the nodal location as the amplitude of the velocity $\frac{d y}{d t}$ is nearly zero here. Note that the effect of structural damping on the location of the node points of an Euler-Bernoulli beam under resonant forcing for a similar physical system is investigated and found to be negligible in Reference. ${ }^{31}$ The power:

$$
P_{t}(t)=F_{t} \frac{d y}{d t}=\left(k(z-y)+c\left(\frac{d z}{d t}-\frac{d y}{d t}\right)\right) \frac{d y}{d t}
$$

transmitted through the anchors as a function of time is presented in Fig. 9(c). The amplitude of the power transmitted is a function of the attachment location $x_{0}$. Figures $8(\mathrm{~b})$, (c) and (d) present the variation in the magnitudes of the force $F_{t}$, the velocity of the anchor $\frac{d y}{d t}$, and the power transmitted through the anchor $P_{t}$, as the anchoring location $x_{0}$ is varied from $x_{0}=0$ to $x_{0}=1$. There are two points along the length of the substrate where the amplitude of the power transmitted through the anchor approaches zero. The first point $x_{0}=0$ is a clamped boundary of the substrate and the second is the location $x_{0}=0.5692$ which is a node point for the assembly of the resonator and the substrate. It is interesting to note the occurrence of a sharp peak (labeled ' $M$ ' on Fig. 8(d)). If the resonator is attached at the location corresponding to the peak, the frequency of forcing which corresponds to the second mode of vibration in this case is maximum and the power transmitted has greatest amplitude.

It is observed that attaching a resonator at a nodal location minimizes the power transferred and the structural interaction between the resonator and the substrate. Therefore, to achieve the expected performance metrics of high Q-factor vibratory sensors, it is recommended to locate resonators at the node points of the assembly.

\section{SUMMARY}

Nodal positioning helps to minimize anchor losses and therefore enhances the overall Q-factor of high performance resonators. This work discusses the problem of positioning resonators at the nodal locations of a substrate. The technique developed is demonstrated in the problem of positioning a single-DoF spring-mass resonator on a generic cantilever substrate. The equations governing the dynamics of the system are presented and the associated eigenvalue problem is solved. The loci of natural frequencies as a function of the resonator location are developed and the nodal locations are identified. The nodal locations are calculated numerically from the characteristic equation as well. A chart depicting the number of possible nodal locations for various values of resonator parameters is presented. For certain resonator parameters, multiple nodal locations exists along the substrate. It was found that there also are additional resonator parameter combinations without a possible nodal location anywhere along the span of the substrate. The nodal locations for various combinations of resonator mass and resonator stiffness are presented in the form of lookup tables. The ideas presented here can be extended to more complicated structures. The method employed is expected to assist designers who aim to position a high performance resonator on a substrate without compromising its Q-factor.

\section{REFERENCES}

1 Chikovani, V., Yatsenko, Y. A., Barabashov, A., Marusyk, P., Umakhanov, E., and Taturin, V. Improved accuracy metallic resonator CVG. IEEE Aerospace and Electronic Systems Magazine, 24(5), 40-43, 2009. https://dx.doi.org/10.1109/MAES.2009.5109952.

${ }^{2}$ Luo, Y., Pan, Y., Zhou, G., Qu, T., Luo, H., and Zhang, B. Annealing Experiments on the Quality Factor of Fused Silica Cylindrical Shell Resonator. In 2019 IEEE International Symposium on Inertial Sensors and Systems (INERTIAL), 1-2. IEEE, 2019.

3 Galliou, S., Goryachev, M., Abbé, P., Vacheret, X., Tobar, M. E., and Bourquin, R. Quality Factor Measurements of Various Types of Quartz Crystal Resonators Op- 
erating Near 4 K. IEEE transactions on ultrasonics, ferroelectrics, and frequency control, 63(7), 975-980,, 2016. https://dx.doi.org/10.1109/tuffc.2015.2508812.

${ }^{4}$ Acar, C. and Shkel, A. MEMS vibratory gyroscopes: structural approaches to improve robustness. Springer Science \& Business Media, 2008.

5 Abdelmoneum, M. A., Demirci, M. U., and Nguyen, C. Stemless wine-glass-mode disk micromechanical resonators. In The Sixteenth IEEE Annual International Conference on Micro Electro Mechanical Systems. Kyoto,, 2003. https://dx.doi.org/10.1109/memsys.2003.1189845.

${ }^{6}$ Lin, Y., Lee, S., Li, S., Xie, Y., Ren, Z., and Nguyen, C. Series-resonant VHF micromechanical resonator reference oscillators. IEEE Journal of Solid-State Circuits, 39(12), 2477-2491,, 2004. https://dx.doi.org/10.1109/jssc.2004.837086.

${ }^{7}$ Binder, D. S., Quevy, E., Koyama, T., Govindjee, S., Demmel, J. W., and Howe, R. T. Anchor loss simulation in resonators. In The Eighteenth IEEE Annual International Conference on Micro Electro Mechanical Systems. Miami,, 2005. https://dx.doi.org/10.1109/memsys.2005.1453885.

${ }^{8}$ Park, Y. and Park, K. C. High-fidelity modeling of MEMS resonators. Part I. Anchor loss mechanisms through substrate. Journal of Microelectromechanical Systems, 13(2), 238-247,, 2004. https://dx.doi.org/10.1109/jmems.2004.825300.

9 Hao, Z., Erbil, A., and Ayazi, F. An analytical model for support loss in micromachined beam resonators with in-plane flexural vibrations. Sensors and Actuators A: Physical, 109(1-2), 156-164,, 2003. https://dx.doi.org/10.1016/j.sna.2003.09.037.

${ }^{10}$ Fuller, C. C., Elliott, S., and Nelson, P. A. Active control of vibration. Academic Press, 1996.

11 Bergman, L. and Nicholson, J. Forced vibration of a damped combined linear system. Journal of Vibration, Acoustics, Stress, and Reliability in Design, 107(3), 275281, 1985. https://dx.doi.org/10.1115/1.3269257.

12 Kukla, S. and Posiadala, B. Free vibrations of beams with elastically mounted masses. Journal of Sound and Vibration, 175(4), 557-564,, 1994. https://dx.doi.org/10.1006/jsvi.1994.1345.

${ }^{13}$ Rossit, C. A. and Laura, P. A. A. Free vibrations of a cantilever beam with a spring - mass system attached to the free end. Ocean Engineering, 28(7), 933-939, 2001. https://dx.doi.org/10.1016/S0029-8018(00)00055-X.

14 Banerjee, J. R. Free vibration of beams carrying spring-mass systems - A dynamic stiffness approach. Computers \& Structures, 104, 21-26, 2012. https://dx.doi.org/10.1016/j.compstruc.2012.02.020.
${ }^{15}$ Lin, H. Y. and Tsai, Y. C. Free vibration analysis of a uniform multi-span beam carrying multiple spring-mass systems. Journal of Sound and Vibration, 302(3), 442-456, 2007. https://dx.doi.org/10.1016/j.jsv.2006.06.080.

16 Yesilce, Y., Demirdag, O., and Catal, S. Free vibrations of a multi-span Timoshenko beam carrying multiple spring-mass systems. Sadhana, 33(4), 385-401, 2008. https://dx.doi.org/10.1007/s12046-008-0026-1.

17 Qiao, H., Li, Q., and Li, G. Vibratory characteristics of flexural non-uniform Euler-Bernoulli beams carrying an arbitrary number of spring-mass systems. International Journal of Mechanical Sciences, 44(4), 725-743, 2002. https://dx.doi.org/10.1016/S0020-7403(02)00007-3.

${ }^{18}$ Cha, P. and Pierre, C. Imposing nodes to the normal modes of a linear elastic structure. Journal of Sound and Vibration, 219(4), 669-687, 1999. https://dx.doi.org/10.1006/jsvi.1998.1914.

${ }^{19}$ Cha, P. Specifying nodes at multiple locations for any normal mode of a linear elastic structure. Journal of Sound and Vibration, 250(5), 923-934, 2002. https://dx.doi.org/10.1006/jsvi.2001.3964.

${ }^{20}$ Cha, P. D. and Rinker, J. M. Enforcing nodes to suppress vibration along a harmonically forced damped Euler-Bernoulli beam. Journal of $\mathrm{Vi}$ bration and Acoustics, 134(5), 051010, 2012. https://dx.doi.org/10.1115/1.4006375.

21 Tian, X. An inverse mode problem for the continuous beam. Inverse Problems in Science and Engineering, 24(7), 1205-1214, 2016. https://dx.doi.org/10.1080/17415977.2016.1160388.

22 Mir Hosseini, F. and Baddour, N. A structured approach to solve the inverse eigenvalue problem for a beam with added mass. Mathematical Problems in Engineering, 2014, 1-13, 2014.

${ }^{23}$ Ghaffari, S., Ng, E. J., Ahn, C. H., Yang, Y., Wang, S., Hong, V. A., and Kenny, T. W. Accurate modeling of quality factor behavior of complex silicon MEMS resonators. Journal of Microelectromechanical Systems, 24(2), 276-288,, 2015. https://dx.doi.org/10.1109/jmems.2014.2374451.

24 Bernstein, J. J., Bancu, M. G., Bauer, J. M., Cook, E. H., Kumar, P., Newton, E., Nyinjee, T., Perlin, G. E., Ricker, J. A., Teynor, W. A., et al. High Q diamond hemispherical resonators: fabrication and energy loss mechanisms. Journal of Micromechanics and Microengineering, 25(8), 085006,, 2015. https://dx.doi.org/10.1088/0960$1317 / 25 / 8 / 085006$. 
Table 9. Comparison of frequencies obtained with existing literature for parameter $x_{0}=1$.

\begin{tabular}{|c|c|c|c|c|c|}
\hline \multirow[b]{2}{*}{ Parameters } & \multirow[b]{2}{*}{ Author(s) } & \multicolumn{4}{|c|}{ Frequency } \\
\hline & & $\omega_{1}$ & $\omega_{2}$ & $\omega_{3}$ & $\omega_{4}$ \\
\hline \multirow[b]{3}{*}{$m=0.2, k=0.1$} & Rossit and Laura $^{13}$ & 0.6952 & 3.5747 & 22.044 & 61.701 \\
\hline & Banerjee $^{14}$ & 0.69517 & 3.5747 & 22.044 & 61.7 \\
\hline & Present & 0.6952 & 3.5747 & 22.0431 & 61.7002 \\
\hline \multirow[b]{3}{*}{$m=1, k=1$} & Rossit and Laura ${ }^{13}$ & 0.8594 & 4.0711 & 22.1257 & 61.7293 \\
\hline & Banerjee $^{14}$ & 0.8592 & 4.0711 & 22.126 & 61.73 \\
\hline & Present & 0.8594 & 4.0712 & 22.1254 & 61.7295 \\
\hline \multirow[b]{3}{*}{$m=1, k=10$} & Rossit and Laura $^{13}$ & 1.4194 & 7.4469 & 22.9987 & 62.0267 \\
\hline & Banerjee $^{14}$ & 1.4194 & 7.447 & 22.999 & 62.027 \\
\hline & Present & 1.4194 & 7.4488 & 22.9993 & 62.0268 \\
\hline
\end{tabular}

25 Zotov, S. A., Simon, B. R., Prikhodko, I. P., Trusov, A. A., and Shkel, A. M. Quality factor maximization through dynamic balancing of tuning fork resonator. IEEE Sensors Journal, 14(8), 2706-2714,, 2014. https://dx.doi.org/10.1109/jsen.2014.2314614.

26 Khine, L. and Palaniapan, M. High-Q bulk-mode SOI square resonators with straight-beam anchors. Journal of Micromechanics and Microengineering, 19(1), 015017,, 2008. https://dx.doi.org/10.1088/0960-1317/19/1/015017.

27 Leissa, A. W. and Qatu, M. S. Vibrations of Continuous Systems. McGraw-Hill, New Delhi,, 2011.

28 Meirovitch, L. Computational Methods in Structural Dynamics. Springer Science \& Business Media, 1980.

29 Hagedorn, P. and DasGupta, A. Vibrations and Waves in Continuous Mechanical Systems. John Wiley \& Sons, England, 2007. https://dx.doi.org/10.1002/9780470518434.

30 Meirovitch, L. Fundamentals of vibrations. McGraw-Hill, New Delhi,, 2001.

31 Geist, B. and McLaughlin, J. R. The effect of structural damping on nodes for the Euler-Bernoulli beam: a specific case study. Applied Mathematics Letters, 7(3), 51-55,, 1994. https://dx.doi.org/0893-9659(94)90112-0.

\section{APPENDIX 1: BENCHMARKING}

Several researchers have investigated the problem of evaluating the natural frequencies of a cantilever beam carrying a mass mounted elastically at its free end. To validate the solution procedure employed in this work, the natural frequencies of this system were obtained using the procedure presented in Section 3 and are compared with those of existing literature in Table 9. The results are in excellent agreement. Note that in Reference, ${ }^{13}$ the author presents the values of parameter $\beta_{i}$ which need to be squared to obtain the natural frequencies being compared.

\section{APPENDIX 2: CRITERION TO LOCATE RESONATORS WHEN MULTIPLE NODAL LOCATIONS EXISTS}

The mode shapes and natural frequencies of the assembly are obtained from the eigenvalue problem presented in
Eq. (9). When multiple nodal locations exists (as for the case presented in Fig. 3), to pick one over the other, it is required to compare the eigenvectors (corresponding to the eigenvalues of magnitude $\sqrt{\frac{k}{m}}$ ) of these cases. Let the eigenvector corresponding to a nodal location $x_{0 q}^{c}$ be $\underline{u}_{q}=$

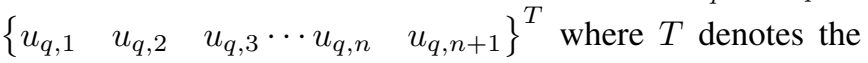
transpose operation. The first $n$ elements of this eigenvector correspond to the mode shape of the substrate and the $(n+1)$ th element to the displacement $z$ of resonator mass. To compare distinct eigenvectors for the same resonator amplitude, these eigenvectors are normalized with respect to the $(n+1)$ th element. Therefore the $z$-normalized mode shapes are obtained as:

$$
\psi_{q}(x)=\frac{1}{u_{q, n+1}} \sum_{i=1}^{n} u_{q, i} \cdot \phi_{i}(x) .
$$

The $z$-normalized mode shapes are those configurations of the substrate which correspond to the same $z$ displacement of magnitude unity. The most energy efficient mounting location out of all possible $x_{0}^{c}$ is that for which the total energy associated with the oscillatory motion of the substrate at the corresponding $z$-normalized mode shape is minimum. The motion of the substrate at such a mode shape with the frequency $\Omega=\sqrt{\frac{k}{m}}$ can be expressed as:

$$
u(x, t)=\psi(x) e^{j \Omega t} .
$$

The total energy associated with the cantilever beam is the sum of its kinetic $(T)$ and potential $(V)$ energies and can be expressed as:

$$
\begin{aligned}
E_{\text {substrate }}= & T_{\text {substrate }}+V_{\text {substrate }}= \\
& \int_{0}^{1} \frac{1}{2}\left(\frac{\partial u}{\partial t}\right)^{2} d x+\int_{0}^{1} \frac{1}{2}\left(\frac{\partial^{2} u}{\partial x^{2}}\right)^{2} d x .
\end{aligned}
$$

Therefore the energy associated with a normalized mode shape (of Eq. (A.2)) of the substrate is:

$$
E_{\text {substrate }}=\frac{1}{2} \int_{0}^{1}\left(-\frac{k}{m} \psi^{2}+\left(\frac{\partial^{2} \psi}{\partial x^{2}}\right)^{2}\right) d x .
$$

Out of all the possible nodal points $x_{0}^{c}$, it is recommended to select the location for which the associated $z$-normalized mode shape returns a minimum value for the integral presented in Eq. (A.4). 